\title{
Melatonin Attenuates Myocardial Ischemia/Reperfusion Injury by Inhibiting Autophagy Via an AMPK/mTOR Signaling Pathway
}

\author{
Wei Ren Chen ${ }^{a} \quad$ Hong Bin Liu ${ }^{a} \quad$ Yun Dai Chen ${ }^{b} \quad$ Yuan Sha ${ }^{a} \quad$ Qiang Mab $^{b}$ \\ Ping Jun Zhu ${ }^{\mathrm{b}}$ Yang $\mathrm{Mu}^{\mathrm{b}}$ \\ aDepartment of Cardiology, Nanlou Division, Chinese PLA General Hospital; National Clinical Research \\ Center for Geriatric Diseases, Beijing, 'bepartment of Cardiology, Chinese PLA General Hospital, \\ Beijing, China
}

\section{Key Words}

Melatonin • Ischemia/reperfusion injury • Autophagy $•$ AMPK $・$ mTOR

\begin{abstract}
Background/Aims: Melatonin has been demonstrated to protect cardiac microvascular endothelial cells (CMECs) against ischemia/reperfusion injury (IRI). Autophagy plays different roles in the heart during ischemia and reperfusion. The AMP activated protein kinase/ mammalian target of rapamycin (AMPK/mTOR) pathway is associated with autophagy. This study sought to explore whether melatonin regulates CMEC autophagy through the AMPK/ mTOR signaling pathway. Methods: The effects of melatonin in IRI were investigated in vivo rat models and in vitro neonatal CMECs. Myocardial infarct size was achieved by Evans blue and triphenyltetrazolium chloride staining. The severity of cell injury was evaluated by cell vitality and lactate dehydrogenase (LDH) release assays, and autophagy was evaluated by transmission electron microscopy and the assessment of autophagy-related gene expression, such as that of Beclin 1 and light chain 3-II. Results: In vivo, melatonin markedly reduced infarcted area, improved cardiac function and decreased LDH release. However, the AMPK activator AICAR and the mTOR inhibitor rapamycin reduced the protective effects of melatonin on IRI. In vitro, Beclin1 and light chain 3-II protein were found to be down-regulated and autophagosomes were found to be reduced in response to melatonin, together with an increase in cell vitality and a decrease in LDH. Treatment with AICAR or rapamycin ablated the benefit observed with melatonin treatment. Conclusions: Melatonin played an important and protective role in CMECs by inhibiting autophagy against IRI via the AMPK/mTOR system.
\end{abstract}

Wei Ren Chen and Yuan Sha contributed equally to this work.

Hong Bin Liu

and Yun Dai Chen 
Chen et al.: Melatonin on Autophagy

\section{Introduction}

Cardiac microvascular endothelial cells (CMECs) are the predominant cell type damaged immediately after myocardial ischemia/reperfusion injury (IRI) [1]. Autophagy plays different roles in the heart during ischemia and reperfusion [2]. The AMP activated protein kinase/mammalian target of rapamycin (AMPK/mTOR) pathway is associated with autophagy [3, 4]. Melatonin is the main indoleamine produced by the pineal gland; it is a well-known antioxidant and free radical scavenger [5]. Several studies have shown that melatonin protects against IRI $[6,7]$. In our previous study, melatonin protected CMECs against oxidative stress injury by activation of the MAPK/ERK signaling pathway [8]. The aim of the present study was to investigate whether melatonin regulates CMEC autophagy through the AMPK/mTOR signaling pathway.

\section{Materials and Methods}

\section{Animal models of cardiac IRI}

Sprague-Dawley (SD) rats (250-300 g) were purchased from the Experimental Animal Center of PLA General Hospital, Beijing, China (Approval No. SCXK 20120001). All animal procedures and experiments were conducted in accordance to the National Institutes of Health Guide for the Care and Use of Laboratory Animals. SD rats were anesthetized with $10 \%$ chloral hydrate before endotracheal intubation. IRI was induced by ligating the left anterior descending artery (LAD) as previously reported [9]. Thirty rats were randomized into five groups: (i) sham group (thoracotomy without ligation); (ii) control group (subjected to ischemia for $30 \mathrm{~min}$ and reperfusion for $2 \mathrm{~h}$ (IRI)); (iii) melatonin group (treated with melatonin $(20 \mathrm{mg} / \mathrm{kg}$ i.p.) $12 \mathrm{~h}$ before IRI) [10].; (iv) melatonin +5 -aminoimidazole-4-carboxamide-1- $\beta$-d-ribofuranoside (AICAR) group (melatonin $(20 \mathrm{mg} / \mathrm{kg}$ i.p.) and AICAR ( $0.7 \mathrm{mg} / \mathrm{g}$ i.p.) $12 \mathrm{~h}$ before IRI) [11].; (v) melatonin + rapamycin group (treated with melatonin ( $20 \mathrm{mg} / \mathrm{kg}$ i.p.) and rapamycin (1mg/kg i.p.) $12 \mathrm{~h}$ before IRI) [12]. At the end of the reperfusion period, echocardiography was performed by echocardiogram (14.0 MHz, Sequoia C512; Acuson, Germany). Hearts were removed and stained with $2 \%$ Evans blue and $1 \%$ triphenyltetrazolium chloride. Infarct size was expressed as percentage of the total left ventricular (LV) area (n=6/group).

\section{Lactate dehydrogenase release}

Lactate dehydrogenase (LDH) is a fairly stable enzyme that is released from the cytosol into the culture medium as a consequence of cellular integrity damage. We used an LDH assay (Beyotime Institute of Biotechnology, Shanghai, China) to evaluate the presence of cell injury or damage.

\section{CMEC isolation, culture and in vitro IRI assay}

CMECs were isolated from the hearts of neonatal SD rats (age: 5-7 days, weight: 12-16 g) using an enzyme dissociation method described in our previous study [8]. Cultured cell purity was evaluated using morphological characteristics and immunohistochemistry. CMEC monolayers displayed uniform 'cobblestone' morphology and demonstrated positive (>95\%) staining for factor VIII (Abcam, \#ab61910) and CD31 (Xinxingtang Biotechnology, \#Xt-0468R) by immunohistochemistry [13]. In vitro IRI was simulated by 30 min hypoxia with serum starvation followed by $2 \mathrm{~h}$ of reoxygenation [14]. Hypoxic conditions were created by replacing culture medium with Hanks solution and exposing the cells to $95 \%$ $\mathrm{N}_{2}$ and $5 \% \mathrm{CO}_{2}$. The pH was adjusted to 6.8 with lactate to mimic ischemic conditions. For drug treatment, cultured CMECs were pre-incubated with $1 \mu \mathrm{M}, 5 \mu \mathrm{M}$ or $10 \mu \mathrm{M}$ melatonin for $12 \mathrm{~h}$ before inducing IRI. To evaluate whether the AMPK/mTOR pathway was involved in the protective effect of melatonin, CMECs were treated with AICAR $(0.5 \mathrm{mM}) 24 \mathrm{~h}$ before inducing IRI or with rapamycin $(50 \mathrm{ng} / \mathrm{mL})$ addition $30 \mathrm{~min}$ before inducing IRI $[14,15]$. The MTT assay was used to evaluate cell viability. Absorbance was measured at 450 nm using a microtiter plate reader (Bio-Rad, USA). Cellular ATP levels were determined using an ATP Assay Kit (Beyotime Institute of Biotechnology, China) according to the manufacturer's protocol [16].

\section{Western blots}

Following experimental treatment, CMECs were lysed with RIPA lysis buffer (Beyotime, China) for 30 $\mathrm{min}$ and centrifuged at 14, $000 \times \mathrm{g}$ for $30 \mathrm{~min}$. Equal amounts of protein were loaded into wells of a $10 \%$ 


\section{Cellular Physiology Cell Physiol Biochem 2018;47:2067-2076 \\ \begin{tabular}{l|l} 
DOI: 10.1159/000491474 & and Biochemistry \\
Published online: July 04, 2018 & $\begin{array}{l}\text { 2018 The Author(s). Published by S. Karger AG, Basel } \\
\text { www.karger.com/cpb }\end{array}$
\end{tabular} \\ Chen et al.: Melatonin on Autophagy}

sodium dodecyl sulfate-polyacrylamide gel. Proteins were separated by gel electrophoresis and transferred to a polyvinylidene difluoride membrane (Millipore, MA, USA). Membranes were blocked with 5\% milk in Tris-buffered saline containing 0.05\% Tween 20 (TBST) at room temperature for $1 \mathrm{~h}$ followed by overnight incubation at $4^{\circ} \mathrm{C}$ with the following primary antibodies: anti-Beclin 1 (1:1000), anti-light chain 3 (LC3) I/II (1:1000), anti-AMPK (1:1000), anti-phospho-AMPK- $\alpha$ (1:1000), anti-mTOR (1:1000), anti-phospho-mTOR (1:500) and anti- $\beta$-actin (1:1000). After overnight incubation, membranes were washed with TBST and further incubated with the appropriate secondary antibody at $37^{\circ} \mathrm{C}$ for $60 \mathrm{~min}$. Membranes were developed with an enhanced chemiluminescence reagent.

\section{Quantitative real-time polymerase chain reaction ( $q R T-P C R$ )}

Total RNA was extracted using TRIzol reagent (Invitrogen) and reverse transcribed with the one-step RT-PCR kit (TransGen Biotech Co., Ltd., China) according to the manufacturer's instructions. The primers used for polymerase chain reaction were as follows: Beclin 1, forward - 5'-GGCAGTGGCTCCTATT-3' ${ }^{\prime}$, reverse - 5'-GGCGTGCTGTGCTCTGAAAA-3'; $\beta$-actin, forward - 5'-GATGGTGGGTATGGGTCAGAAGGAC-3', reverse 5'-GCTCATTGCCGATAGTGATGACT-3'. Quantification of gene expression was performed using the ABI PRISM 7500 Sequence Detection System (Applied Biosystems, Foster City, CA) with SYBR Green (TransGen Biotech Co., Ltd., China). mRNA levels were determined by qRT-PCR in triplicate for each of the independently prepared RNAs and were normalized to $\beta$-actin expression.

Detection of autophagosomes by transmission electron microscopy

Transmission electron microscopy, the standard method to detect autophagy, was used to further clarify the effects of melatonin on autophagy. CMECs were collected, fixed with $2.5 \%$ glutaraldehyde in 0.1 M phosphate buffer at $4^{\circ} \mathrm{C}$ for $2 \mathrm{~h}$ then post-fixed in $1 \%$ osmic acid at $4^{\circ} \mathrm{C}$ for $1.5 \mathrm{~h}$. Cells were washed with PBS, dehydrated by a serial gradient ethanol and embedded in Embed-812 medium (Electron Microscopy Sciences). The resulting blocks were cut into ultrathin sections using an Ultramicrotome (Leica, Reichert Ultracuts) then stained with $0.2 \%$ lead citrate/1\% uranyl acetate. The ultrastructure of the cells was then observed under a transmission electron microscope (JEM1230; JEOL, Japan).

\section{Statistical analysis}

Data are described as the mean \pm standard deviation (SD) of at least three independent experiments and were analyzed by one-way analysis of variance (ANOVA). The limit of statistical significance between treated and control groups was $\mathrm{P}<0.05$.

\section{Results}

Melatonin reverses IRI in vivo

As shown in Fig. 1A, mice treated with melatonin developed smaller infarcts (expressed as a percentage of the total LV). However, the beneficial effects were not observed when melatonin was used with AICAR or rapamycin. LDH release was significantly increased in response to IRI and melatonin significantly reduced IRI-induced LDH release. Melatonin improved cardiac function in the context of IRI as evidenced by an improvement in left ventricular ejection fraction (LVEF). However, AICAR and rapamycin reduced the protective effects of melatonin on cardiac function (Fig. 1B).

\section{Beclin $1 \mathrm{mRNA}$ and the ratio of LC3-II/I protein in IRI were downregulated by melatonin} in vivo

Beclin1 mRNA was upregulated in the infarcted myocardium after IRI, however, this IRIinduced upregulation was blocked in the group of rats that were pretreated with melatonin (Fig. 1C). After IRI, the ratio of LC3-II/I protein in the infarcted myocardium of the control group was significantly increased (vs. non-IRI rats). In the group pre-treated with melatonin, this ratio was significantly lower compared with the control group. However, treatment with AICAR or rapamycin ablated the benefit observed with melatonin treatment (Fig. 1D). 


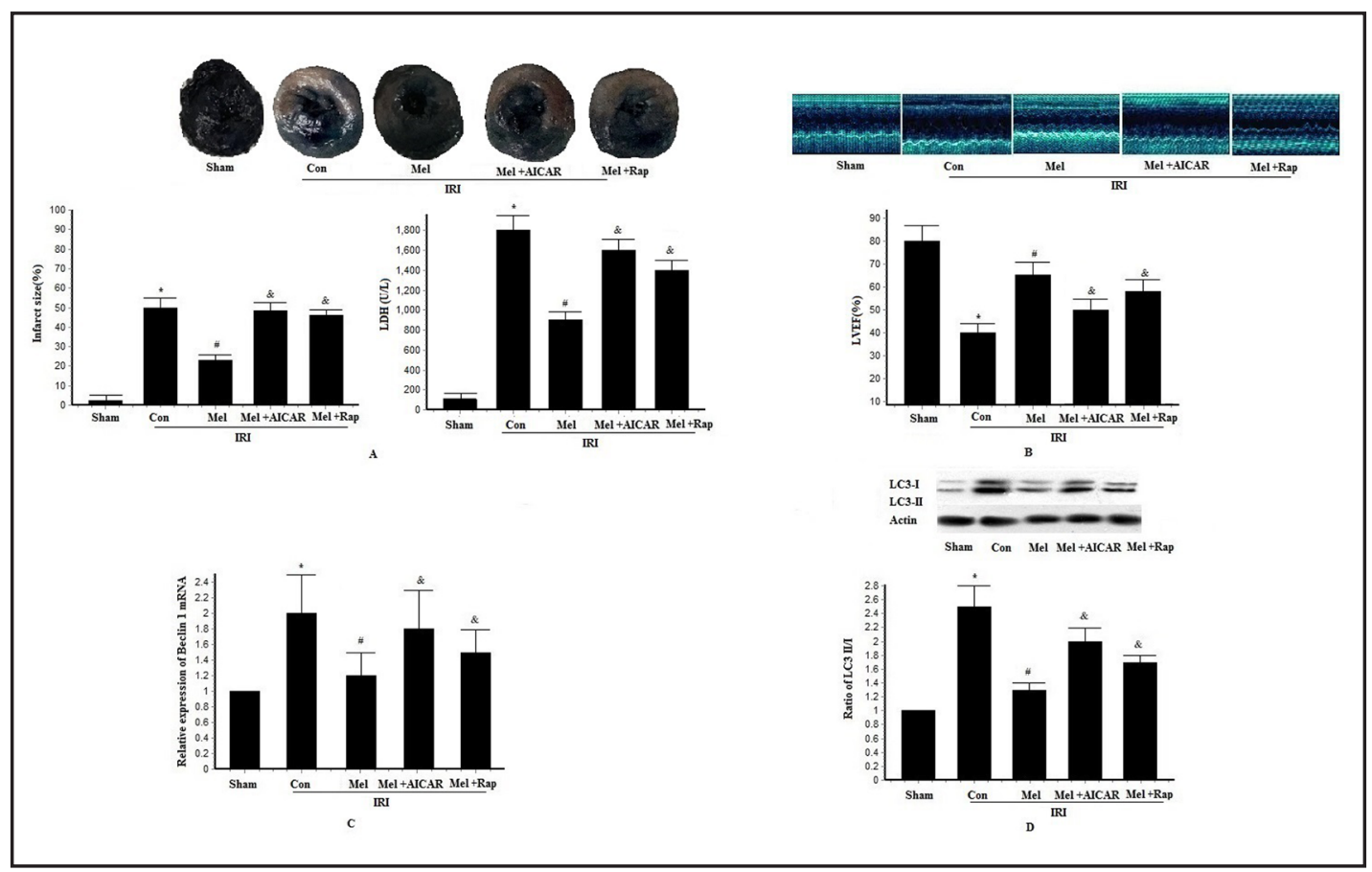

Fig. 1. Melatonin reduced infarct size and preserved cardiac function following ischemia/reperfusion injury in vivo which was performed by 30 min of ischemia followed by $2 \mathrm{~h}$ reperfusion ( $n=6)$. (A) Results of infarct size and lactate dehydrogenase (LDH). (B) Result of left ventricular ejection fraction (LVEF). (C) Result of Beclin1 mRNA expression (infarcted myocardium). (D) Results of LC3-I and LC3-II protein expression, and the ratio of LC3-II/I was calculated to demonstrate the autophagy level (infarcted myocardium). ${ }^{*} \mathrm{P}<0.05$ vs Sham, \# $\mathrm{P}<0.05$ vs Con, $\& \mathrm{P}<0.05$ vs Mel.

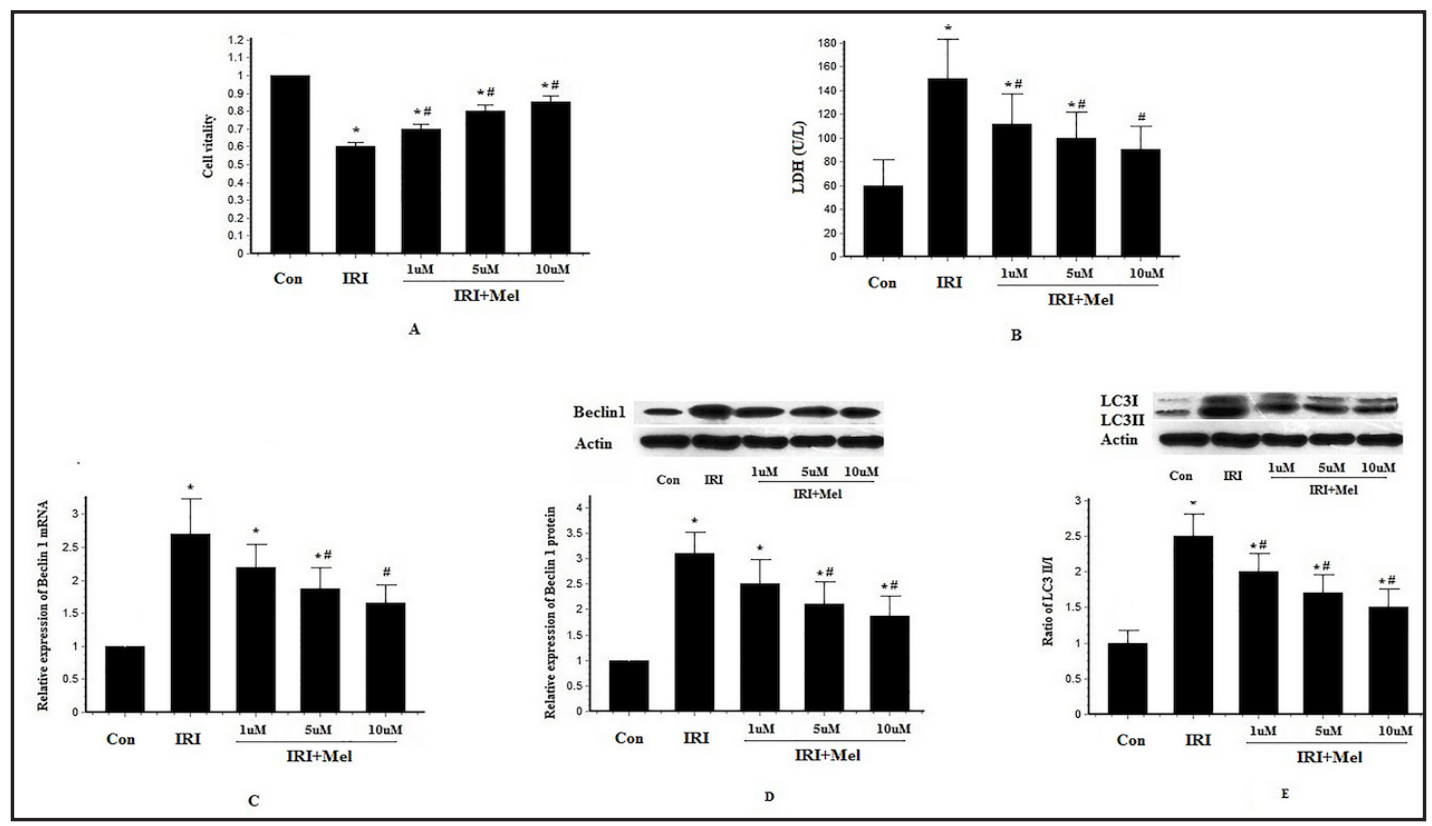

Fig. 2. Effects of melatonin on cell vitality and autophagy in CMECs $(n=3)$. (A) Cell vitality was measured with MTT method. (B) LDH level of culture serum. (C) Result of Beclin1 mRNA expression. (D) Result of Beclin1 protein expression. (E) Results of LC3-I and LC3-II protein expression. ${ }^{*} \mathrm{P}<0.05$ vs Con, \# $\mathrm{P}<0.05$ vs IRI.

\section{KARGER}




\section{Melatonin treatment attenuated IRI-induced injury in vitro}

CMECs exposed to simulated IRI demonstrated a significant decrease in cell viability and a significant increase in LDH release compared with CMECs not exposed to IRI. To evaluate the protective effect of melatonin, CMECs were treated with melatonin before IRI. Cell viability was increased and LDH release was decreased after IRI in the melatonin treated group compared with the untreated group (Fig. s 2A and 2B). Cell viability in the $10 \mu \mathrm{M}$ melatonin group was not significantly higher than in the $5 \mu \mathrm{M}$ melatonin group $(\mathrm{P}>0.05)$. Therefore, subsequent experiments were performed using melatonin at a concentration of $5 \mu \mathrm{M}$.

\section{IRI induced CMEC autophagy was attenuated by melatonin}

CMECs were subjected to simulated IRI and autophagy was evaluated using autophagosome markers Beclin 1 and LC3-II/I ratio. Beclin 1 mRNA levels were determined by qRT-PCR and protein levels of Beclin 1 and LC3-I and -II were determined by western blotting. The ratio of LC3-II/I was used to measure the relative autophagy level of CMECs. Beclin 1 mRNA and protein along with the ratio of LC3- II/I were found to be increased by IRI in untreated cells but in CMECs treated with melatonin prior to IRI, the levels were decreased relative to the untreated cells (Fig. s 2C-E).

\section{Effect of melatonin on AMPK/mTOR signaling}

Western blot was performed to determine whether melatonin induced the expression and phosphorylation of AMPK and mTOR in CMECs. As shown in Fig. 3A, AMPK and mTOR signaling was detected in CMECs. Melatonin treatment significantly decreased AMPK phosphorylation and increased mTOR phosphorylation without inducing the expression of AMPK or mTOR. The AMPK activator AICAR $(0.5 \mathrm{mM})$ was applied with and without melatonin for $24 \mathrm{~h}$. A significant increase in AMPK phosphorylation was observed, as well as inhibition of the effects of melatonin on the phosphorylation of AMPK and mTOR in the presence of IRI $(\mathrm{P}<0.05)$. We also investigated the effects of rapamycin on the phosphorylation of AMPK. We found that $50 \mathrm{ng} / \mathrm{mL}$ rapamycin activated the phosphorylation of AMPK (Fig. 3B). These data indicate that melatonin modulates the AMPK/mTOR signaling pathway.

\section{Melatonin inhibited CMEC autophagy via AMPK/mTOR activation}

To examine the effect of AMPK or mTOR on CMECs, the cells were pretreated with AICAR or rapamycin, with or without IRI. The ratio of LC3-II/I was increased by AICAR or rapamycin,

Fig. 3. Effects of melatonin on the phosphorylation of AMPK and mTOR in CMECs (n=3). (A) The effects of AICAR on the phosphorylation of AMPK and mTOR. Cells were pre-treated with or without AICAR $(0.5 \mathrm{mM})$ for $24 \mathrm{~h}$ and stimulated with or without melatonin ( $5 \mathrm{uM})$ before IRI $(\mathrm{n}=3) .{ }^{*} \mathrm{P}<0.05$ vs Con (without IRI), $\# \mathrm{P}<0.05$ vs the values of cells treated with melatonin alone, $\Delta \mathrm{P}<0.05$ vs the values of cells after IRI, \&P $<0.05$ vs the values of cells treated with melatonin alone after IRI. (B) The effects of rapamycin on the phosphorylation of AMPK and mTOR $(\mathrm{n}=3) .{ }^{*} \mathrm{P}<0.05$ vs Con (without IRI), $\Delta \mathrm{P}<0.05$ vs the values of cells after IRI.

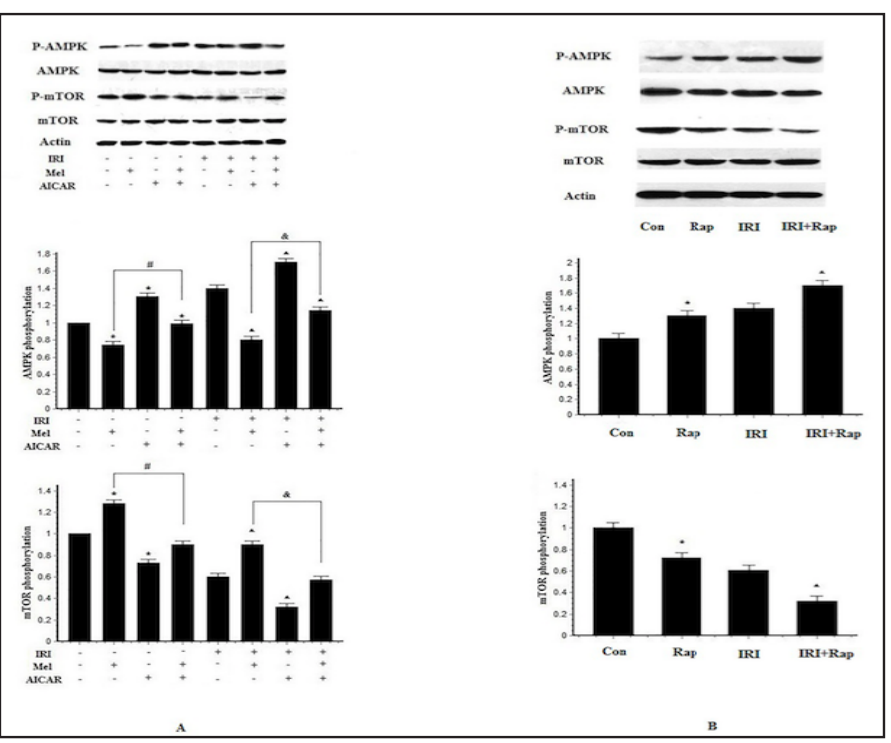



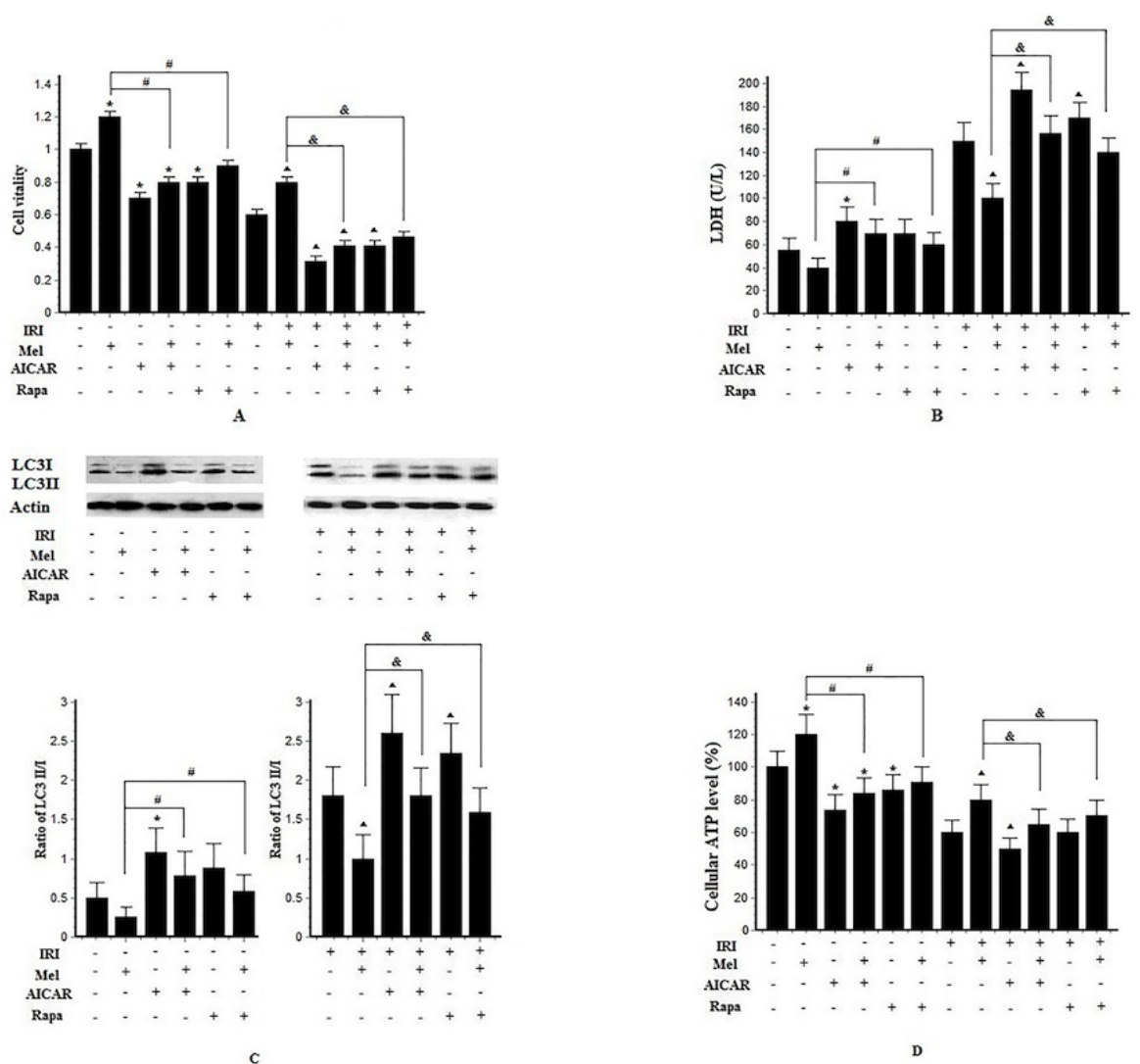

Fig. 4. The effects of melatonin and AMPK/mTOR pathway inhibitors on cell vitality, autophagy and cellular ATP levels in CMECs (n=3). (A) Cell vitality was measured with MTT method. (B) LDH level of culture serum. (C) Comparing with melatonin group, the ratio of LC3-II/I was increased by AICAR or rapamycin. (D) Result of cellular ATP level. ${ }^{*} \mathrm{P}<0.05$ vs Con (without IRI), \#P<0.05 vs the values of cells treated with melatonin alone, $\Delta \mathrm{P}<0.05$ vs the values of cells after IRI, $\& \mathrm{P}<0.05$ vs the values of cells treated with melatonin alone after IRI.

together with an increase in LDH release and a decrease in cell viability (without IRI). To demonstrate whether melatonin inhibited CMEC autophagy via AMPK or mTOR, the CMECs were treated with AICAR or rapamycin before IRI. In that experiment, LDH was increased and cell viability was decreased compared with the melatonin group. In addition, the ratio of LC3- II/I was increased by AICAR or rapamycin, which attenuated the inhibitory effect of melatonin on autophagy (with IRI) (Fig. 4A-C). Autophagosomes observed by transmission electron microscopy were significantly reduced by melatonin (Fig. 5).

\section{Effects of melatonin and AMPK/mTOR pathway inhibitors on CMEC ATP levels}

AMPK is a metabolic regulator that balances net energy production with the prevailing metabolic demands in eukaryotic cells. AMPK is activated by a decrease in intracellular ATP levels under different stress conditions (e.g., glucose deprivation, hypoxia, ischemia) [17]. Our experiments demonstrated that melatonin increased cellular ATP levels after IRI. AICAR and rapamycin inhibited the melatonin induced increase in cellular ATP levels. These results suggest that the effect of melatonin on cellular ATP levels might be dependent on AMPK/ mTOR signaling (Fig. 4D). 


\section{Cellular Physiology Cell Physiol Biochem 2018;47:2067-2076

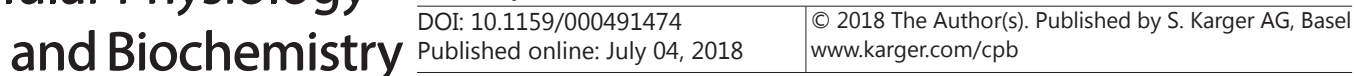 Chen et al.: Melatonin on Autophagy}

Fig. 5. The effects of melatonin and AMPK/ mTOR pathway inhibitors on autophagosomes. (A) Autophagosomes were observed by transmission electron microscope without IRI. (B) Autophagosomes were increased after IRI. (C) Autophagosomes were decreased by melatonin. (D) The inhibitory effect of melatonin on autophagy was attenuated by AICAR. (E) The inhibitory effect of melatonin on autophagy was attenuated by rapamycin. Magnification, $\times 30000$. Red arrows indicate autophagosomes.

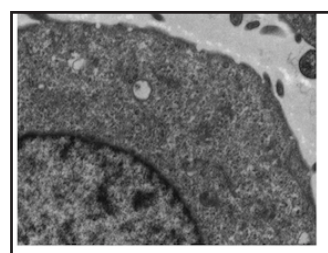

(A) Con

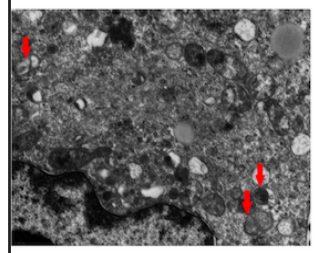

(C) IRI+Mel

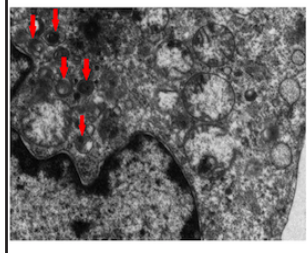

(E) IRI+Mel+Rap

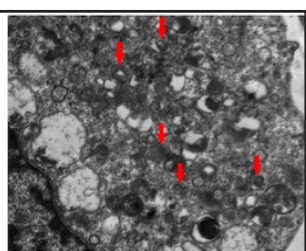

(B) IRI

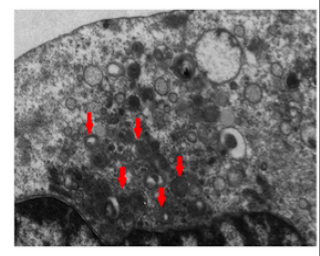

(D) IRI+Mel+AICAR

\section{Discussion}

In the present study, we investigated the effects of melatonin on CMEC autophagy after IRI and the molecular mechanism by which these effects were produced. Our results suggest that the decrease of CMEC autophagy by melatonin is mediated, at least in part, by AMPK/ mTOR signaling.

Autophagy has been demonstrated to be involved in many physiological and pathological processes. The effect of melatonin on autophagy has been investigated more recently [1820]. Yoo and Jeung showed that melatonin suppresses CsA-mediated autophagy in rat pituitary GH3 cells [18]. San-Miguel et al. demonstrated that melatonin inhibits autophagy and endoplasmic reticulum stress in mice with carbon tetrachloride-induced fibrosis [19].

Autophagy is promoted by AMPK, which is a key energy sensor and regulates cellular metabolism to maintain energy homeostasis [21]. Conversely, autophagy is inhibited by mTOR, a central cell-growth regulator that integrates growth factor and nutrient signals [22]. Xie et al. found that melatonin ameliorates cardiac hypertrophy caused by chronic intermittent hypoxia by inducing autophagy via AMPK activation [23]. However, Kwon et al. showed that melatonin treatment inhibited AMPK activity and exerted synergistic effects on their neuroprotective properties [24]. The phosphorylation of AMPK was blocked by treatment with melatonin and the AMPK- activating compound, AICAR, induced neuronal cell death in HT22 hippocampal cells. Our experiments demonstrated that melatonin increased cell viability and inhibited the phosphorylation of AMPK. Furthermore, we used AICAR to enhance the phosphorylation of AMPK. We found that AICAR reduced the effects of melatonin on AMPK phosphorylation and inhibited the effects of melatonin on CMEC autophagy in the context of IRI response. Our data suggest that melatonin inhibits CMEC autophagy through AMPK signaling.

A study by Behram et al. showed that melatonin may have a significant protective effect on primordial follicles via activation of the mTOR pathway [25]. The findings of Kang, et al. suggest that melatonin downregulates autophagy via activation of mTOR signaling which may in turn contribute to its protective effects in liver IRI [26]. Our data show that melatonin activated mTOR. The AMPK/mTOR pathway antagonists, AICAR and rapamycin, 


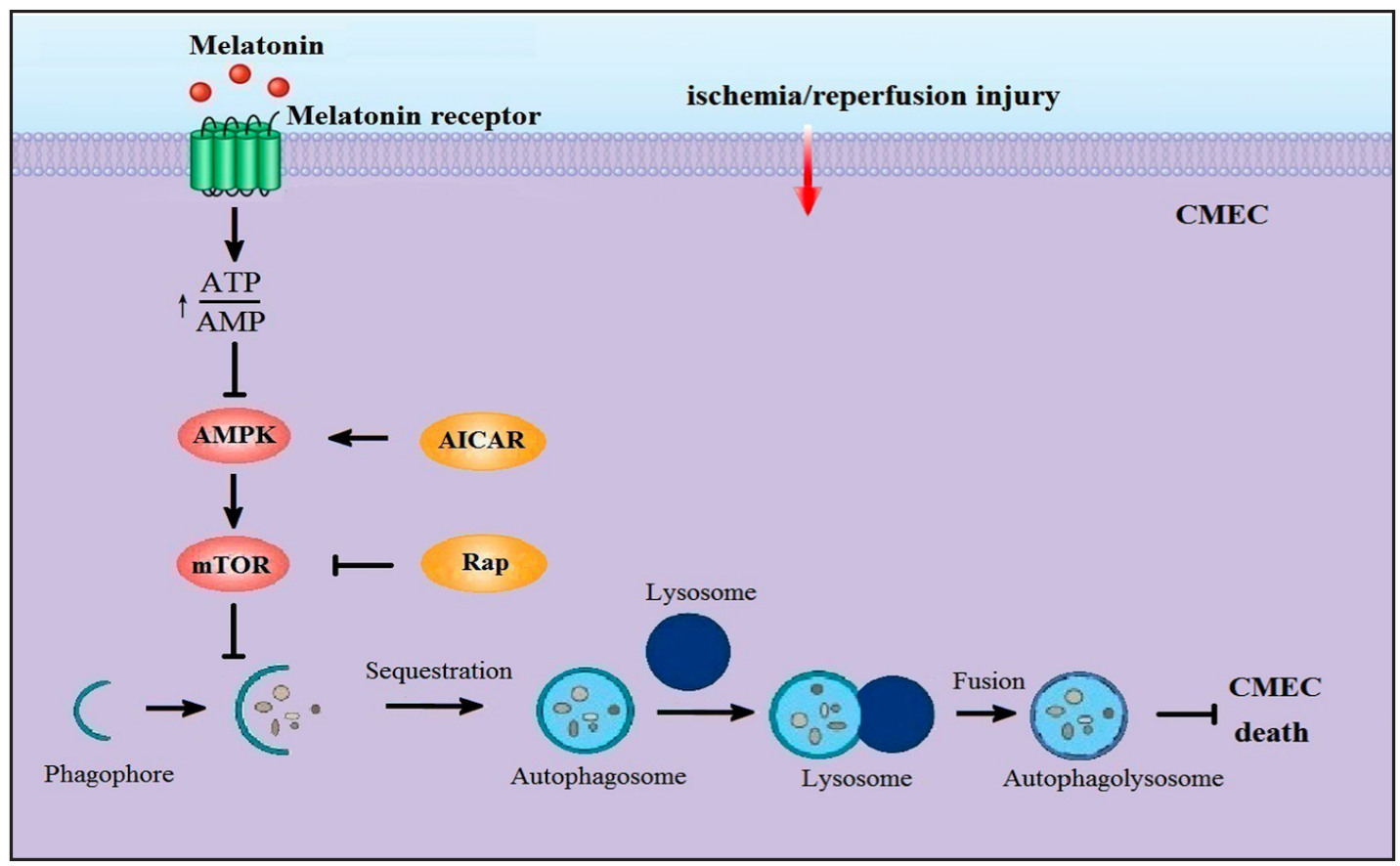

Fig. 6. Schematic representation showing that melatonin inhibited CMEC autophagy through an AMPK/ mTOR signaling pathway. Melatonin increased the ATP/AMP ratio, which in turn inhibited AMPK and subsequently activated mTOR. These effects subsequently inhibited CMEC autophagy.

decreased the melatonin-induced phosphorylation of mTOR and increased CMEC autophagy. Taken together, melatonin increased the ATP/AMP ratio, which in turn inhibited AMPK and subsequently activated mTOR. These effects subsequently inhibited CMEC autophagy (Fig. 6).

\section{Conclusion}

In summary, our study demonstrated that melatonin played an important and protective role in CMECs by inhibiting autophagy against IRI via the AMPK/mTOR system.

\section{Acknowledgements}

We express our sincere appreciation to all participants in this study. We also thank Li Yi and Jie Liu, who assisted in this study. All authors have substantially contributed to the manuscript in terms of conception and design, analysis and interpretation of data, drafting the article, revising it critically for important intellectual content, and final approval of the version. This study was supported by China Cardiovascular Association V.G Clinical Research Fund (fund number 2017-CCA-VG-035) and the National Natural Science Foundation of China (fund number 81441008).

\section{Disclosure Statement}

The authors declared no potential conflicts of interest with respect to the research, authorship, or publication of this article. 


\section{Cellular Physiology Cell Physiol Biochem 2018;47:2067-2076 \begin{tabular}{l|l} 
DOI: 10.1159/000491474 & $\begin{array}{l}\text { O 2018 The Author(s). Published by S. Karger AG, Basel } \\
\text { www.karger.com/cpb }\end{array}$ \\
\hline
\end{tabular}}

Chen et al.: Melatonin on Autophagy

\section{References}

1 Wei L, Sun D, Yin Z, Yuan Y, Hwang A, Zhang Y, Si R, Zhang R, Guo W, Cao F, Wang H: A PKC-beta inhibitor protects against cardiac microvascular ischemia reperfusion injury in diabetic rats. Apoptosis 2010;15:488-498.

2 Zhao Y, Zhang L, Qiao Y, Zhou X, Wu G, Wang L, Peng Y, Dong X, Huang H, Si L, Zhang X, Zhang L, Li J, Wang W, Zhou L, Gao X: Heme oxygenase-1 prevents cardiac dysfunction in streptozotocin-diabetic mice by reducing inflammation, oxidative stress, apoptosis and enhancing autophagy. PLoS One 2013;8:e75927.

- 3 Fujihara S, Morishita A, Ogawa K, Tadokoro T, Chiyo T, Kato K, Kobara H, Mori H, Iwama H, Masaki T: The angiotensin II type 1 receptor antagonist telmisartan inhibits cell proliferation and tumor growth of esophageal adenocarcinoma via the AMPK $\alpha /$ mTOR pathway in vitro and in vivo. Oncotarget 2017;8:85368549.

4 Wu ST, Sun GH, Cha TL, Kao CC, Chang SY, Kuo SC, Way TD: CSC-3436 switched tamoxifen-induced autophagy to apoptosis through the inhibition of AMPK/mTOR pathway. J Biomed Sci 2016;23:60.

5 Chovancova B, Hudecova S, Lencesova L, Babula P, Rezuchova I, Penesova A, Grman M, Moravcik R, Zeman M, Krizanova O: Melatonin-Induced Changes in Cytosolic Calcium Might be Responsible for Apoptosis Induction in Tumour Cells. Cell Physiol Biochem 2017;44:763-777.

-6 Cui Y, Ren L, Li B, Fang J, Zhai Y, He X, Du E, Miao Y, Hua J, Peng S: Melatonin Relieves Busulfan-Induced Spermatogonial Stem Cell Apoptosis of Mouse Testis by Inhibiting Endoplasmic Reticulum Stress. Cell Physiol Biochem 2017;44:2407-2421.

7 Liu L, Xu Y, Reiter RJ, Pan Y, Chen D, Liu Y, Pu X, Jiang L, Li Z: Inhibition of ERK1/2 Signaling Pathway is Involved in Melatonin's Antiproliferative Effect on Human MG-63 Osteosarcoma Cells. Cell Physiol Biochem 2016;39:2297-2307.

-8 Zhu H, Jin Q, Li Y, Ma Q, Wang J, Li D, Zhou H, Chen Y: Melatonin protected cardiac microvascular endothelial cells against oxidative stress injury via suppression of IP3R-[Ca2+]c/VDAC-[Ca2+]m axis by activation of MAPK/ERK signaling pathway. Cell Stress Chaperones 2018;23:101-113.

-9 He B, Xiao J, Ren AJ, Zhang YF, Zhang H, Chen M, Xie B, Gao XG, Wang YW: Role of mir-1 and mir-133a in myocardial ischemic postconditioning. J Biomed Sci 2011;18:22.

10 Han D, Huang W, Li X, Gao L, Su T, Li X, Ma S, Liu T, Li C, Chen J, Gao E, Cao F: Melatonin facilitates adiposederived mesenchymal stem cells to repair the murine infarcted heart via the SIRT1 signaling pathway. J Pineal Res 2016;60:178-192.

11 Kristiansen SB, Solskov L, Jessen N, Løfgren B, Schmitz O, Nielsen-Kudsk JE, Nielsen TT, Bøtker HE, Lund S: 5-Aminoimidazole-4-carboxamide-1-beta-D-ribofuranoside increases myocardial glucose uptake during reperfusion and induces late pre-conditioning: potential role of AMP-activated protein kinase. Basic Clin Pharmacol Toxicol 2009;105:10-16.

-12 Temiz-Resitoglu M, Kucukkavruk SP, Guden DS, Cecen P, Sari AN, Tunctan B, Gorur A, Tamer-Gumus L, Buharalioglu CK, Malik KU, Sahan-Firat S: Activation of mTOR/IкB- $\alpha / \mathrm{NF}-\kappa B$ pathway contributes to LPSinduced hypotension and inflammation in rats. Eur J Pharmacol 2017;802:7-19.

13 Zhang Z, Li W, Sun D, Zhao L, Zhang R, Wang Y, Zhou X, Wang H, Cao F: Toll-like receptor 4 signaling in dysfunction of cardiac microvascular endothelial cells under hypoxia/reoxygenation. Inflamm Res 2011;60:37-45.

-14 Xiao J, Zhu X, Kang B, Xu J, Wu L, Hong J, Zhang Y, Ni X, Wang Z: Hydrogen Sulfide Attenuates Myocardial Hypoxia-Reoxygenation Injury by Inhibiting Autophagy via mTOR Activation. Cell Physiol Biochem 2015;37:2444-2453.

15 Miao XY, Gu ZY, Liu P, Hu Y, Li L, Gong YP, Shu H, Liu Y, Li CL: The human glucagon-like peptide-1 analogue liraglutide regulates pancreatic beta-cell proliferation and apoptosis via an AMPK/mTOR/P70S6K signaling pathway. Peptides 2013;39:71-79.

16 Liu B, Tang M, Han Z, Li J, Zhang J, Lu P, Song N, Wang Z, Yin C, Zhang W: Co-incubation of human spermatozoa with anti-VDAC antibody reduced sperm motility. Cell Physiol Biochem 2014;33:142-150.

17 Cai Y, Wang Q, Ling Z, Pipeleers D, McDermott P, Pende M, Heimberg H, Van de Casteele M: Akt activation protects pancreatic beta cells from AMPK-mediated death through stimulation of mTOR. Biochem Pharmacol 2008;75:1981-1993.

18 Yoo YM, Jeung EB: Melatonin suppresses cyclosporine A-induced autophagy in rat pituitary GH3 cells. J Pineal Res 2010;48:204-211. 


\section{Cellular Physiology Cell Physiol Biochem 2018;47:2067-2076 \begin{tabular}{l|l} 
DOI: 10.1159/000491474 & $\begin{array}{l}\text { O 2018 The Author(s). Published by S. Karger AG, Basel } \\
\text { www.karger.com/cpb }\end{array}$ \\
\hline
\end{tabular} \\ Chen et al.: Melatonin on Autophagy}

19 San-Miguel B, Crespo I, Sánchez DI, González-Fernández B, Ortiz de Urbina JJ, Tuñón MJ, González-Gallego J: Melatonin inhibits autophagy and endoplasmic reticulum stress in mice with carbon tetrachloride-induced fibrosis. J Pineal Res 2015;59:151-162.

20 Chen Y, Zhang J, Zhao Q, Chen Q, Sun Y, Jin Y, Wu J: Melatonin Induces Anti-Inflammatory Effects to Play a Protective Role via Endoplasmic Reticulum Stress in Acute Pancreatitis. Cell Physiol Biochem 2016;40:1094-1104.

-21 Pyo JO, Nah J, Jung YK: Molecules and their functions in autophagy. Exp Mol Med 2012;44:73-80.

-22 Egan DF, Shackelford DB, Mihaylova MM, Gelino S, Kohnz RA, Mair W, Vasquez DS, Joshi A, Gwinn DM, Taylor R, Asara JM, Fitzpatrick J, Dillin A, Viollet B, Kundu M, Hansen M, Shaw RJ: Phosphorylation of ULK1 (hATGl) by AMP-activated protein kinase connects energy sensing to mitophagy. Science 2011;331:456461.

-23 Xie S, Deng Y, Pan YY, Wang ZH, Ren J, Guo XL, Yuan X, Shang J, Liu HG: Melatonin protects against chronic intermittent hypoxia-induced cardiac hypertrophy by modulating autophagy through the 5' adenosine monophosphate-activated protein kinase pathway. Biochem Biophys Res Commun 2015;464:975-981.

24 Kwon KJ, Kim HJ, Shin CY, Han SH: Melatonin Potentiates the Neuroprotective Properties of Resveratrol Against Beta-Amyloid-Induced Neurodegeneration by Modulating AMP-Activated Protein Kinase Pathways. J Clin Neurol 2010;6:127-137.

25 Behram Kandemir Y, Aydin C, Gorgisen G: The effects of melatonin on oxidative stress and prevention of primordial follicle loss via activation of mTOR pathway in the rat ovary. Cell Mol Biol (Noisy-le-grand) 2017;63:100-106.

-26 Kang JW, Cho HI, Lee SM: Melatonin inhibits mTOR-dependent autophagy during liver ischemia/ reperfusion. Cell Physiol Biochem 2014;33:23-36. 